“C 2017 IEEE. Personal use of this material is permitted. Permission from IEEE must be obtained for all other uses, in any current or future media, including reprinting/republishing this material for advertising or promotional purposes, creating new collective works, for resale or redistribution to servers or lists, or reuse of any copyrighted component of this work in other works." 


\section{Optimal Strict Frequency Reuse in Cellular Networks-based Stochastic Geometry Model}

\author{
Sinh Cong Lam \\ University of Technology, Sdyney \\ Center of Real Time Information Network \\ Faculty of Engineering and Information Technology \\ Email: SinhCong.Lam@student.uts.edu.au
}

\author{
Kumbesan Sandrasegaran \\ University of Technology, Sdyney \\ Center of Real Time Information Network \\ Faculty of Engineering and Information Technology \\ Email:Kumbesan.Sandrasegaran@uts.edu.au
}

\begin{abstract}
This paper studies Strict Frequency Reuse algorithm which can be utilised to mitigate the InterCell Interference as well as improve the spectrum efficiency in cellular networks by dividing the users into two groups called Cell-Center Users (CCUs) and Cell-Edge Users (CEUs). In this paper, we defined a two-phase operation of FR for both $\mathrm{CCU}$ and $\mathrm{CEU}$ in which each user is classified as either CCU or CEU during the first phase and data transfer between the user and it's servng Base Station during the second phase. The performance of the user, i.e. CCU and CEU, as well as the network system in Strict Frequency Reuse network with multi-users and multi-Resource Blocks (RBs) are derived. The analytical results which are verified by Monte Carlo simulation indicate that when SNR or SINR threshold increases, the system performance reaches the peak before steadily decreasing. Thus, the optimal value of SNR and SINR threshold are selected to maximise the performance of the network system.
\end{abstract}

Index Terms: Poisson Cellular Network, coverage probability, throughput, strict frequency reuse, Rayleigh fading.

\section{INTRODUCTION}

The rapid development of Internet-connected mobile subscribers as well as the demand for broadband services have driven the urgent requirement for the network operators to work on a new network architecture. Long Term Evolution (LTE) cellular system has been developed by 3rd Generation Partnership Project (3GPP) as a next cellular network generation. In a LTE network, the dense frequency reuse will be used to allow two adjacent cells to use the same frequency band. However, the reuse frequency with a high density leads to the increase of InterCell Interference (ICI) which limits the performance of mobile users, especially for Cell-Edge users (CEUs) who experience low Signal-toInterference-plus-Noise ratios (SINRs). InterCell Interference Coordination (ICIC) technique has been introduced to control the reuse of frequencies as well as related network parameters such as transmit power in order to mitigate ICI.

Strict Frequency Reuse (Strict FR) algorithm is the basic ICIC technique that divides the allocated Resource Blocks (RBs) into $\Delta+1$ groups including one Cell-Center (CC) RB group or common RB group and $\Delta$ Cell-Edge (CE) RB groups or private RB groups. $\Delta$ is called FR Factor. Each cell is allocated a CC RB group and a CE RB group. The CC RBs are assigned to CC Users (CCUs), whose SINRs are greater than a pre-determined SINR threshold. The CE RBs are assigned to CE Users (CEUs) whose initial SINRs are smaller than the SINR threshold. Generally, the operation of Strict FR algorithm can be divided into two phases. During the first phase, based on the reported SINR, the BSs classify the users into CCUs and CEUs. During the second phase, the communication between the users and the BSs is established and data is transferred.

\section{Power}

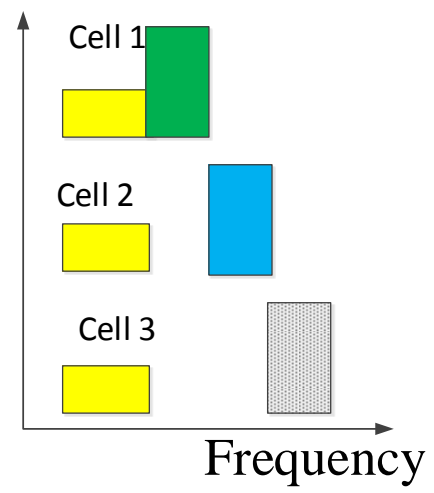

Fig. 1: Strict Frequency Reuse algorithm

In the literature, the performance analysis of Soft FR frequently has been presented for a hexagonal network model by using network-level simulation [1]. Since the deployments of cellular networks depend on a large number of practical conditions such as transmit environment and user distribution, research work based on stochastic geometry models to analyse the network performance [2]-[4] is becoming more popular in the literature. In most works, the Poisson Point Process (PPP) in which the BSs are distributed as Spatial Poisson Point Process and the cell boundaries are followed by Voronoi diagram has been selected to replace the regular hexagonal model and to provide mathematical tractability. Although, the PPP network model has been utilised as early as 1997 in [5], the first important results about the performance of frequency 
reuse algorithm were presented in 2011 [6]. In these papers, the performance of the network was presented as average coverage probability and data rate. Although, reference [6] presented the basis of the analysis of frequency reuse, the impact of the number of users on the system performance was not addressed. In [7], the performance of the Strict FR algorithm was evaluated and optimised for the case of multi users with Round Robin scheduling. However, this work assumed that the BSs utilise the same power to serve all users that implies that there is no difference between the CCU and CEU.

In [8], the performance of a typical user which can be served as a CCU or a CEU was presented, and the optimal design parameter of SINR threshold for frequency reuse algorithms was analysed. Although, the power channel gain of the CEU changed every phase, it was assumed that the CCU experiences the same channel power gain during two phases. Furthermore, the numbers of users as well as RBs were not discussed.

Compared with the previous work in [6], [9], our works proposed new following approaches

- We defition a two-phase operation for both CCU and CEU. In addtion, the density of interfering BSs cause interference to CEU is only $\frac{\lambda}{\Delta}$ in which $\lambda$ is the denstiy of BSs in the network and $\Delta$ is the FR factor.

- The number of CCUs and CEUs are analysed base on the intial state of the network.

- The number of RBs are considered to evaluate the network performance.

Furtherore, the impact of the parameters of Strict FR algorithm on the network performance are addressed and thus the optimal SNR and SINR threshold are analysed. At the initial state, it is assumed that there is one user in each area of each cell. The number of new users entering to network is assumed to be a Poisson random variable. During the first phase, each new user is assumed to associate with the nearest BS where it is classified as a CCU or CEU based on the received SINR and a SINR threshold. The number of CCUs and CEUs for different values of SINR threshold and Signal-to-Noise (SNR) are presented. During the second phase, both CEU and CCU experience new SINRs. The performance formulations of users such as the average coverage probability and capacity are presented as the conditional probabilities of the second phase under condition of the first phase.

\section{SYSTEM MODEL}

\section{A. Network topology}

We consider a PPP cellular network is characterised by the density of BSs $\lambda$, the standard transmit power $P$. The downlink signals are assumed to experience Rayleigh channel power gain $g$ and path loss with exponent $\alpha$.

Under this network model, the open access protocol in which a user is allowed to associate with any BS is studied. However, the user prefers a connection with the BS which has the greatest average signal power. The average signal power of the user at a distance $r$ from a BS is defined as:

$$
\bar{P}=\operatorname{Pr}^{-\alpha} E[g]
$$

in which $E(g)$ is the expected value of channel power gain $g$. In this paper, it is assumed that the channel power gain are i.i.d random variable and $E[g]=1$.

For single-tier networks, all BSs transmit on CC RBs at the same power and the path loss exponent is constant. Hence, the association problem can be formulated as:

$$
\operatorname{Pr}^{-\alpha}=\max \left(P_{j} r_{j}^{-\alpha}\right) \quad \text { or } \quad r<r_{j} \quad \forall 0<j \leq K
$$

in which $K$ is the total number of BSs in the network.

Thus, the Probability Density Function (PDF) of the distance $r$ between the user and its serving BS is given by [2]:

$$
f_{R}(r)=2 \pi \lambda r e^{-\lambda \pi r^{2}}
$$

It is assumed that at initial state, there are $M^{(o)}$ users at a typical cell. The number of new users who request connections to the network is assumed to be a random Poisson variable with mean $\lambda^{(u)}$. We denote $S$ as the area of the PPP network. The total number of new users are $\lambda^{(u)} S$ while the number of cells is $\lambda S$. Hence, the average number of new users per cell is given by:

$$
M^{(n)}=\frac{\lambda^{(u)}}{\lambda}
$$

Consequently, the number of users at the typical cell can be obtained by

$$
M=M^{(o)}+M^{(n)}
$$

\section{B. Frequency Reuse Algorithm}

In this paper, all cells are assumed to use Strict FR with the same reuse factor $\Delta$ as shown in Figure 1. For example, the resource allocation technique uses the SINR threshold $T$ to classify $M$ users in each cell into $M^{(c)}$ CCUs and $M^{(e)}$ CEUs, $N$ Resource Blocks (RBs) into $N^{(c)}$ CC RBs and $N^{(e)}$ CE RBs. Furthermore, the $\mathrm{CC} \mathrm{RBs}$ are used as the common resources for all CCUs while the CE RBs is divided into $\Delta$ private $\mathrm{RB}$ groups. Since, the CCUs do not share their resources with the CEUs, each group of CEUs is allocated $N^{(e)} / \Delta$ RBs.

1) InterCell Interference: In a cellular network system, it is assumed that the scheduler randomly allocates a RB from the available RBs to a user. To represent use of $\mathrm{RB} b$, an indicator function $\tau\left(R B^{(z)}=b\right)$ that take values 1 if $\mathrm{RB} b$ is used in area $z$ of a cell is defined in which $z=c$ or $z=e$ corresponds to a $\mathrm{CC}$ or CE Area.

The expected value of the indication function is called as the resource allocation ratio and given by:

$$
\varepsilon^{(z)}=E\left[\tau\left(R B^{(z)}=b\right)\right]= \begin{cases}1 & \text { if } M^{(z)}>N^{(z)} \\ \frac{M^{(z)}}{N^{(z)}} & \text { if } M^{(z)}<N^{(z)}\end{cases}
$$

It is assumed that the typical user is served on $\mathrm{RB} b$. We denote $\theta^{(c)}$ and $\theta^{(e)}$ as the set of interfering BSs transmitting on the $\mathrm{CC}$ and $\mathrm{CE}$ RBs. Hence, the densities of BSs in $\theta^{(c)}$ 
and $\theta^{(e)}$ are $\lambda^{(c)}=\lambda$ and $\lambda^{(e)}=\lambda / \Delta[10]$. The ICI of a typical CCU and CEU are respectively given by:

$$
\begin{aligned}
& I_{S t r}^{(c)}=\sum_{z_{c} \in \theta^{(c)}} \tau\left(R B^{(c)}=b\right) \tau\left(R B^{\left(z_{c}\right)}=b\right) P g_{z_{c}} r_{z_{c}}^{-\alpha} \\
& I_{S t r}^{(e)}=\sum_{z_{e} \in \theta^{(e)}} \tau\left(R B^{(e)}=b\right) \tau\left(R B^{z_{e}}=b\right) \phi P g_{z_{e}} r_{\left(z_{e}\right)}^{-\alpha}
\end{aligned}
$$

in which $g_{z}$ and $r_{z}$ are the channel power gain and distance from the user to the interfering BS $z, z=\left\{z_{c}, z_{e}\right\}$ correspond to $\mathrm{CC}$ and $\mathrm{CE}$ Area.

\section{Instantaneous SINR}

In the Strict FR network system, the typical user can be served on the CE RB with a higher transmit power (CE power )or on the $\mathrm{CC} \mathrm{RB}$ with a lower transmit power (CC power). Hence, the received SINR of a typical user from its serving $\mathrm{BS}$ in tier $k$ can be expressed by the following equation:

$$
\begin{cases}\operatorname{SINR}(\phi, r)=\frac{\phi P g r^{-\alpha}}{I_{S t r}^{(e)}+\sigma^{2}} & \text { for CEU } \\ \operatorname{SINR}(1, r)=\frac{P g r^{-\alpha}}{I_{S t r}^{(c)}+\sigma^{2}} & \text { for CCU }\end{cases}
$$

in which $g$ and $r$ is the channel power gain and distance from the user to the serving BS; $\phi$ is the transmit power ratio; $\sigma^{2}$ is Gaussian noise; $I_{S t r}^{(c)}$ and $I_{S t r}^{(e)}$ are the powers of ICIs and defined in $7 \mathrm{a}$ and $7 \mathrm{~b}$.

To represent the network parameters at initial state where there are $M^{(o)}$ users in the network, a letter $o$ is added into the upper index of each parameter. For example, the ICIs for a CCU and CEU at this state are denoted by $I_{S t r}^{(o c)}$ and $I_{S t r}^{(o e)}$; the allocation ratios are $\varepsilon^{(o c)}$ and $\varepsilon^{(o e)}$ and the SINR is $\operatorname{SINR}^{(o)}$.

\section{Number of new Cell-Center and Cell-Edge users}

A new user is served as a CCU if its received SINR at the initial state is greater than the SINR threshold. Therefore, the probability in which the user at a distance $r$ from its serving BS is served as a CCU is defined as

$$
\mathbb{P}^{(c)}(T \mid r)=\mathbb{P}\left(\operatorname{SINR}^{(o)}(1, r)>T\right)
$$

in which $\operatorname{SINR}^{(o)}$ is the received SINR of the user at initial state and defined in Equation 8.

Substituting Equation 8 into Equation 9 and denoting $S N R=$ $\frac{P}{\sigma^{2}}, \mathbb{P}^{(c)}(T \mid r)$ is evaluated using following steps:

$$
\begin{aligned}
= & \mathbb{E}\left[\exp \left(-\frac{\operatorname{Tr}^{\alpha}\left(I_{u}^{(o c)}+\sigma^{2}\right)}{P}\right)\right] \\
= & \exp \left(-\frac{T r^{\alpha}}{S N R}\right) \mathbb{E}\left[\exp \left(-\frac{\operatorname{Tr}^{\alpha} I_{u}^{(o c)}}{P}\right)\right] \\
= & \exp \left(-\frac{T r^{\alpha}}{S N R}\right) \\
& \mathbb{E}\left[\prod_{z_{c} \in \theta_{c}} \exp \left(-\tau\left(R B^{(o c)}=b\right) \tau\left(R B_{z_{c}}^{(o c)}=b\right) T g_{z_{c}} \frac{r_{z_{c}}^{-\alpha}}{r^{-\alpha}}\right)\right]
\end{aligned}
$$

$$
\begin{aligned}
& \stackrel{(\mathrm{a})}{=} \exp \left(-\frac{T r^{\alpha}}{S N R}\right) \mathbb{E}\left[\prod_{z_{c} \in \theta_{c}}\left(1-\frac{\varepsilon^{(o) 2} T\left(\frac{r_{z_{c}}}{r}\right)^{-\alpha}}{1+T\left(\frac{r_{z c}}{r}\right)^{-\alpha}}\right)\right] \\
& \stackrel{(\mathrm{b})}{=} \exp \left(-\frac{T r^{\alpha}}{S N R}\right) \exp \left(-2 \pi \lambda \int_{r}^{\infty} \frac{\varepsilon^{(o) 2} T\left(\frac{r_{z c}}{r}\right)^{-\alpha}}{1+T\left(\frac{r_{z c}}{r}\right)^{-\alpha}} r_{z_{c}} d r_{z_{c}}\right) \\
& \stackrel{(\mathrm{c})}{=} \exp \left(-\frac{T r^{\alpha}}{S N R}-\pi \lambda r^{2} f^{(o)}(T)\right)
\end{aligned}
$$

in which (a) follows the Rayleigh fading assumption and definition of allocation ratio in Equation 6; (b) follows the properties of PPP probability generating function [11]; (c) results from the properties of Gamma function and GaussLegendre approximation [12]; and

$$
f^{(o)}(T)=\varepsilon^{(o) 2}\left[\frac{\pi \frac{2}{\alpha} T^{\frac{2}{\alpha}}}{\sin \left(\frac{\pi(\alpha-2)}{\alpha}\right)}-\sum_{n=1}^{N} \frac{c_{n}}{2} \frac{T}{T+\left(\frac{x_{n}+1}{2}\right)^{\frac{\alpha}{2}}}\right]
$$

Therefore, the average number of new CCUs is obtained by

$$
M^{(n c)}(T)=\frac{\lambda^{(u)}}{\lambda} \int_{0}^{\infty} \mathbb{P}^{(c)}(T \mid r) f_{R}(r) d r
$$

in which $f_{R}(r)$ is the PDF of the distance $r$. Thus,

$$
M^{(n c)}(T)=2 \pi \lambda^{(u)} \int_{0}^{\infty} r e^{-\frac{T r^{\alpha}}{S N R}-\pi \lambda r^{2}\left(1+f^{(o)}(T)\right)} d r
$$

and the average number of new CEUs is

$$
M^{(n e)}(T)=\frac{\lambda^{(u)}}{\lambda}-2 \pi \lambda^{(u)} \int_{0}^{\infty} r e^{-\frac{T r^{\alpha}}{S N R}-\pi \lambda r^{2}\left(1+f^{(o)}(T)\right)} d r
$$

\section{Coverage PRobability OF A TYPICAL USER}

\section{A. Average Coverage Probability definition}

Cell-Center User: When the instantaneous received SINR of a user during the first phase is greater than the SINR threshold, the BS does not make any change in neither RB nor transmit power. Due to the changes of the instantaneous channel power gain and of the numbers of CCUs and CEUs between two phases, the new SINR is denoted by $\operatorname{SINR}(1, r)$ and obtained from Equation 8 . The coverage probability for the $\mathrm{CCU}$ are defined as the following conditional probability:

$$
P_{c}^{(c)}(T)=\mathbb{P}(\operatorname{SINR}(1, r)>\hat{T} \mid \operatorname{SINR}(1, r)>T)
$$

Cell-Edge User: when the user is classified as a CEU, it will be served on a CE different RB with CE transmit power. Thus, the user experience new $\operatorname{SINR}, \operatorname{SINR}(\phi, r)$, which is obtained from Equation 8 . Hence, the coverage probability of the CEU is defined as:

$$
P_{c}^{(e)}(T)=\mathbb{P}\left(\operatorname{SINR}(\phi, r)>\hat{T} \mid \operatorname{SINR}^{(o)}(1, r)<T\right)
$$




\section{B. Average Coverage Probability of a new CCU and CEU}

Deploying the same approach given Section II-D, the average coverage probability of a new $\mathrm{CCU}$ is evaluated as follows

$$
\begin{gathered}
P_{c}^{(c)}(T)=\frac{\mathbb{P}\left(\operatorname{SINR}(1, r)>\hat{T}, \operatorname{SINR}^{(o)}(1, r)>T\right)}{\mathbb{P}\left(\operatorname{SINR}^{(o)}(1, r)>T\right)} \\
=\frac{\int_{0}^{\infty} r e^{-\pi \lambda r^{2}} \mathbb{P}\left(\operatorname{SINR}(1, r)>\hat{T}, \operatorname{SINR}^{(o)}(1, r)>T \mid r\right) d r}{\int_{0}^{\infty} r e^{-\pi \lambda r^{2}} \mathbb{P}\left(\operatorname{SINR}^{(o)}(1, r)>T \mid r\right) d r} \\
=\frac{\int_{0}^{\infty} r e^{-\frac{(T+\hat{T}) r^{\alpha}}{\operatorname{SNR}}-\pi r^{2} \lambda\left(1+f^{(o)}(T)+f^{(c)}(\hat{T})\right)} d r}{\int_{0}^{\infty} r e^{-\frac{T r^{\alpha}}{S N R}-\pi r^{2} \lambda\left(1+f^{(o)}(T)\right)} d r}
\end{gathered}
$$

One of the differences between the CCU and CEU is that density of interfering BSs of the CCU is $\lambda$ while that of the $\mathrm{CCU}$ is $\lambda / \Delta$. Furthermore, although the CEU is served with high transmit power, the transmit powers of the serving and interfering BS of the CUE are the same. Thus, the average coverage probability of the CUE is given by

$$
\begin{gathered}
P_{c}^{(e)}(T)=\frac{\mathbb{P}\left(\operatorname{SINR}(\phi, r)>\hat{T}, \operatorname{SINR}^{(o)}(1, r)>T\right)}{\mathbb{P}\left(\operatorname{SINR} R^{(o)}(1, r)<T\right)} \\
=\frac{\int_{0}^{\infty} r e^{-\pi \lambda r^{2} \mathbb{P}\left(\operatorname{SINR}(\phi, r)>\hat{T}, \operatorname{SINR}^{(o)}(1, r)>T \mid r\right) d r}}{\int_{0}^{\infty} r e^{-\pi \lambda r^{2}} \mathbb{P}\left(\operatorname{SINR}^{(o)}(1, r)<T \mid r\right) d r} \\
=\frac{2 \pi \lambda \int_{0}^{\infty} r e^{-\frac{\hat{T} r^{\alpha}}{\phi S N R}-\pi r^{2} \lambda\left(1+\frac{f^{(e)}(\hat{T})}{\Delta}\right)}\left(1-e^{-\frac{T r^{\alpha}}{S N R}-\pi r^{2} \lambda f^{(o)}(T)}\right) d r}{1-2 \pi \lambda \int_{0}^{\infty} r e^{-\frac{T r^{\alpha} \alpha}{S N R}-\pi r^{2} \lambda\left(1+f^{(o)}(T)\right)} d r}
\end{gathered}
$$

\section{Average Coverage Probability of the typical user}

In this section, we derive the average coverage probability of a typical user (called random user) that is randomly located in the network and can be served as a CEU or CCU. Conventionally, each user who requests a connection to the network is classified as a CCU or CEU. Hence, the coverage probability of the user at the distance $r$ from its serving BS is defined by

$$
\begin{aligned}
\mathbb{P}(T \mid r)= & \mathbb{P}(\operatorname{SINR}(1, r)>\hat{T} \mid r) \mathbb{P}\left(\operatorname{SINR}^{(o)}(1, r)>T \mid r\right) \\
& +\mathbb{P}(\operatorname{SINR}(\phi, r)>\hat{T} \mid r) \mathbb{P}\left(\operatorname{SINR}^{(o)}(1, r)<T \mid r\right)
\end{aligned}
$$

Using the result of Section III-B, the coverage of the user is obtained by

$$
\begin{aligned}
\mathbb{P}(T \mid r) & =e^{-\frac{(T+\hat{T}) r^{\alpha}}{S N R}}-\pi r^{2} \lambda\left(f^{(o)}(T)+f^{(c)}(\hat{T})\right) d r \\
& +e^{-\frac{\hat{T}_{r} r^{\alpha}}{S N R}-\frac{\pi r^{2} \lambda}{\Delta} f^{(e)}(\hat{T})}\left(1-e^{-\frac{T r^{\alpha}}{S N R}-\pi r^{2} \lambda f^{(o)}(T)}\right)
\end{aligned}
$$

Hence, the average coverage probability of the typical user is achieved by integrating the conditional probability $\mathbb{P}(T \mid r)$ over the network

$$
\mathbb{P}(T)=\int_{0}^{\infty} \mathbb{P}(T \mid r) f_{R}(r) d r
$$

in which $f_{R}(r)$ is the PDF of the distance $r$ from the user to its serving BS.

\section{Average Cell Throughput}

\section{A. Average data rate of a $C C U$ and $C E U$}

The average data rate of a user with serving SINR, SINR, is defined as

$$
C=\mathbb{E}_{\text {SINR }}[\ln (\operatorname{SINR}+1)]
$$

The user is served as a CEU if the its instantaneous received SINR at the initial state of the network from its serving BS is below the SINR threshold. The average rate of the user $C^{(e)}(T)$ in this case is obtained by the following conditional expectation :

$$
\begin{aligned}
& \mathbb{E}\left[\ln (\operatorname{SINR}(\phi, r)+1)>\gamma \mid \operatorname{SINR}^{(o)}(1, r)<T\right] \\
& =\int_{0}^{\infty} \mathbb{P}\left(\ln (\operatorname{SINR}(\phi, r)+1)>\gamma \mid \operatorname{SINR}^{(o)}(1, r)<T\right) d \gamma
\end{aligned}
$$

Using the same approach given in III-B, the average rate of the CEU $C^{(e)}(T)$ is evaluated as follows:

$$
\begin{aligned}
& =\int_{0}^{\infty} \frac{\mathbb{P}\left(\ln (\operatorname{SINR}(\phi, r)+1)>\gamma, \operatorname{SINR}^{(o)}(1, r)<T\right)}{\mathbb{P}\left(\operatorname{SINR} R^{(o)}(1, r)<T\right)} d \gamma \\
& =\int_{0}^{\infty} \frac{\mathbb{P}\left(\operatorname{SINR}(\phi, r)>e^{\gamma}-1, \operatorname{SINR}^{(o)}(1, r)<T\right)}{\mathbb{P}\left(\operatorname{SINR} R^{(o)}(1, r)<T\right)} d \gamma \\
& =\frac{\pi \lambda \int_{0}^{\infty} \int_{0}^{\infty} e^{-\frac{e^{\gamma}-1}{\phi S N R} t^{\alpha / 2}-\pi \lambda\left(1+\frac{1}{\Delta} f^{(e)}\left(e^{\gamma}-1\right)\right) t} d t d \gamma}{1-\pi \lambda \int_{0}^{\infty} e^{-\frac{T}{S N R} t^{\alpha / 2}-\pi \lambda\left(1+f^{(o)}(T)\right) t} d t} \\
& -\frac{\pi \lambda \int_{0}^{\infty} \int_{0}^{\infty} e^{-\left(\frac{e^{\gamma}-1}{\phi S N R}+\frac{T}{S N R}\right) t^{\alpha / 2}-\pi \lambda\left(1+\frac{1}{\Delta} f^{(e)}\left(e^{\gamma}-1\right)+f^{(o)}(T)\right) t} d t d \gamma}{1-\pi \lambda \int_{0}^{\infty} e^{-\frac{T}{S N R} t^{\alpha / 2}-\pi \lambda\left(1+f^{(o)}(T)\right) t} d t}
\end{aligned}
$$

Similarity, the average data rate of the CCU is given by

$$
\begin{aligned}
C^{(c)}(T) & =\mathbb{E}\left[\ln (\operatorname{SINR}(1, r)+1)>\gamma \mid \operatorname{SINR}^{(o)}(1, r)>T\right] \\
= & \int_{0}^{\infty} \frac{\mathbb{P}\left(\ln (\operatorname{SINR}(1, r)+1)>\gamma, \operatorname{SINR}^{(o)}(1, r)>T\right)}{\mathbb{P}\left(\operatorname{SINR} R^{(o)}(1, r)>T\right)} d \gamma \\
= & \frac{\int_{0}^{\infty} \int_{0}^{\infty} e^{-\frac{e^{\gamma}-1}{S N R} t^{\frac{\alpha}{2}}-\pi \lambda\left(1+f^{(c)}\left(e^{\gamma}-1\right)\right) t} e^{-\frac{T}{S N R} t^{\frac{\alpha}{2}}-\pi \lambda f^{(o)}(T) t} d t d \gamma}{\int_{0}^{\infty} e^{-\frac{T}{S N R} t^{\frac{\alpha}{2}}-\pi \lambda\left(1+f^{(o)}(T)\right) t} d t}
\end{aligned}
$$

\section{B. Average cell data rate}

The average cell throughput is defined as the total data rate of all users in that cell, and can be obtained by:

$$
R=M^{(e)} C^{(e)}(T)+M^{(c)} C^{(c)}(T)
$$

in which $M^{(e)} C^{(e)}(T)$ and $M^{(c)} C^{(c)}(T)$ are average data rate of $\mathrm{CE}$ and CC Area, respectively; $M^{(e)}$ and $M^{(c)}$ are the numbers of CEUs and CCUs connected to the typical cell; $C^{(e)}$ and $C^{(c)}$ 


$$
\begin{aligned}
R=\pi \lambda^{(u)} & \int_{0}^{\infty} \int_{0}^{\infty} e^{-\frac{e^{\gamma}-1}{S N R} t^{\alpha / 2}-\pi \lambda\left(1+f^{(c)}\left(e^{\gamma}-1,1\right)\right) t} e^{-\frac{T}{S N R} t^{\alpha / 2}-\pi \lambda f^{(o)}(T) t} d t d \gamma \\
& +\pi \lambda^{(u)} \int_{0}^{\infty} \int_{0}^{\infty} e^{-\frac{e^{\gamma}-1}{\phi S N R} t^{\alpha / 2}-\pi \lambda\left(1+\frac{1}{\Delta} f^{(e)}\left(e^{\gamma}-1\right)\right) t}\left(1-e^{-\frac{T}{S N R} t^{\alpha / 2}-\pi \lambda f^{(o)}(T) t}\right) d t d \gamma
\end{aligned}
$$

are average data rate of the CEU and CCU which are obtained from Equation 23 and Equation 25. The average cell data rate is given by Equation 24 .

In case of interference-limited network, i.e. $\sigma^{2}=0$, the average capacity of each area is given by

- For Cell-Center Area

$$
\begin{aligned}
R^{(c)} & =M^{(c)} C^{(c)}(T) \\
& =\pi \lambda^{(u)} \int_{0}^{\infty} \int_{0}^{\infty} e^{-\pi \lambda\left(1+f^{(c)}\left(e^{\gamma}-1,1\right)+f^{(o)}(T)\right) t} d t d \gamma \\
& =\frac{\lambda^{(u)}}{\lambda} \int_{0}^{\infty} \frac{1}{1+f^{(c)}\left(e^{\gamma}-1\right)+f^{(o)}(T)} d \gamma
\end{aligned}
$$

- For Cell-Edge Area

$$
\begin{aligned}
& R^{(e)}= M^{(e)} C^{(e)}(T) \\
&= \pi \lambda^{(u)} \int_{0}^{\infty} \int_{0}^{\infty} e^{-\pi \lambda\left(1+\frac{1}{\Delta} f^{(e)}\left(e^{\gamma}-1\right)\right) t} d t d \gamma \\
& \quad-\pi \lambda^{(u)} \int_{0}^{\infty} \int_{0}^{\infty} e^{-\pi \lambda\left(1+\frac{1}{\Delta} f^{(e)}\left(e^{\gamma}-1\right)+f^{(o)}(T)\right) t} d t d \gamma \\
&= \frac{\lambda^{(u)}}{\lambda} \int_{0}^{\infty} \frac{1}{1+\frac{1}{\Delta} f^{(e)}\left(e^{\gamma}-1\right)} d \gamma \\
& \quad-\frac{\lambda^{(u)}}{\lambda} \int_{0}^{\infty} \frac{1}{1+\frac{1}{\Delta} f^{(e)}\left(e^{\gamma}-1\right)+f^{(o)}(T)} d \gamma \quad(28
\end{aligned}
$$

\section{Simulation AND Discussion}

\section{A. Simulation setup}

In the simulation, it is assumed that the network uses Strict FR algorithm with reuse factor of 3 . The network is allocated 50 RBs which are saperated into 40 CC RBs and 10 CE RBs.

Initially, it was assumed that there is one user in each CC and CE Area. The analytical and simulation parameters used in this paper are summarized in Table I.

\begin{tabular}{|l|l|}
\hline Parameters & Value \\
\hline Density of BSs & $\lambda=0.5$ \\
\hline SNR & $10 \mathrm{~dB}$ \\
\hline Transmit power ratio & $\phi=20$ \\
\hline Frequency reuse factor & $\Delta=3$ \\
\hline
\end{tabular}

TABLE I: Analytical and simulation parameters

Figure 2 compares the simulation and the analytical results of the average coverage probabilities of the CCU and CEU for

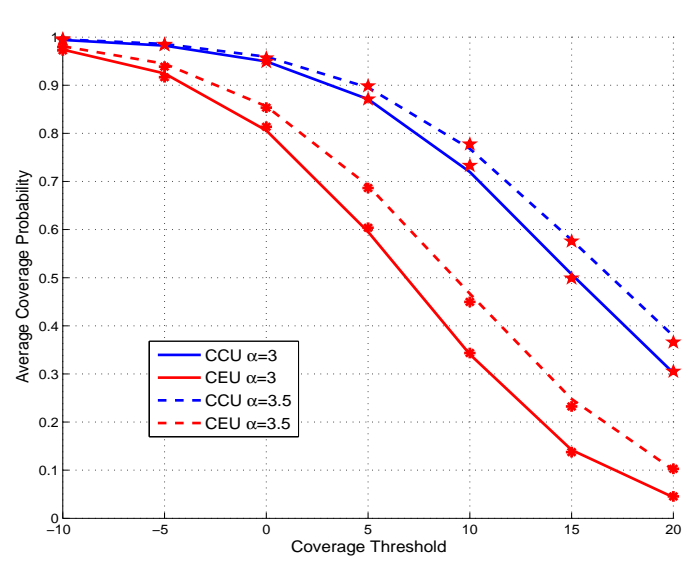

Fig. 2: Average coverage probability of the user with different values of coverage thresholds. The dotted and solid lines represent the analytical results. The star points represent the simulation results.

different values of coverage threshold. It can be observed from the figure that the average coverage probabilities of the $\mathrm{CCU}$ and CEU are inversely proportional to the coverage thresholds. For example, when the coverage threshold increases from $5 \mathrm{~dB}$ to $10 \mathrm{~dB}$ and $\alpha=3$, the average coverage probability decreases by about $57.8 \%$ from 0.8136 to 0.3434 and for the CEU and $23 \%$ from 0.9565 to 0.7332 for the CCU.

\section{B. Optimal SNR}

In this section, the effects of the SNR on the network performance are analysed. It is clear that increasing SNR improves the received signal strength at the user. This leads to an increase in the number of CCUs while the number of CEUs reduces. Since, the numbers of CC and CE BSs are unchanged, the allocation ratio of CC Area which represents the probability that two BSs transmit on the same RB increases while that of CE Area decreases. Consequently, this results in the increase in the average capacity of the CEU and a decrease in the average capacity of the CCU as shown in Figure 3. For example, when the SNR increases $4 \mathrm{~dB}$ from $6 \mathrm{~dB}$ to $10 \mathrm{~dB}$, the average capacity of the CCU decreases by around $37.47 \%$ from 2.599 to $1.625(\mathrm{bit} / \mathrm{s} / \mathrm{Hz})$ while the average capacity of the CEU increases by 26.26\% from 1.504 to 1.899 (bit/s/Hz).

Although the average capacity of the $\mathrm{CCU}$ increases, the rapid decrease in number of CCUs leads to the decrease in the average capacity of CE Area as shown in Figure 4. Hence, the 

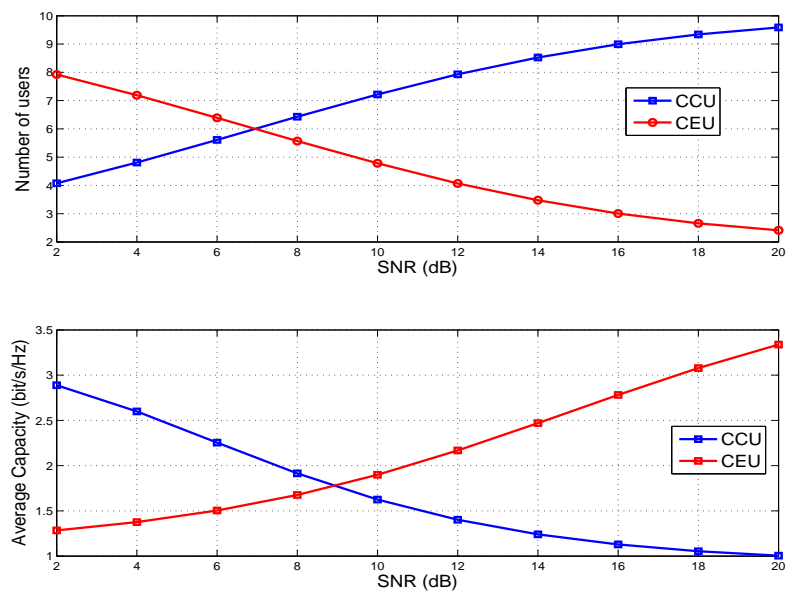

Fig. 3: Average number of CCUs and CCUs and Average data rate of the $\mathrm{CCU}$ and $\mathrm{CEU}$

optimal SNR is selected to achieve high average capacity for both the CCU and CEU as well as cell areas. It can be observed from the figure that the optimal SNR occurs at $S N R=4 d B$ when the average cell capacity peaks at about $22.1(\mathrm{bit} / \mathrm{s} / \mathrm{Hz})$.

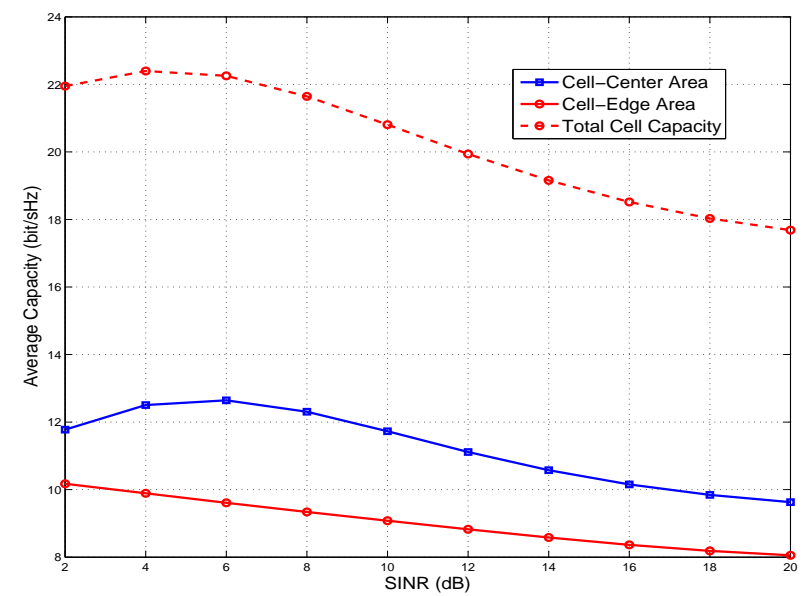

Fig. 4: Average data rate of Cell-Center and Cell-Edge Area

\section{Optimal SINR threshold}

The SINR threshold is used to partition the users into CCUs and CEUs in which the users are defined as CCUs if received SINRs during the first phase are greater than the SINR threshold and the rest of the users are defined as CEUs. Hence, when SINR threshold increases, the number of CCUs increases while that of the CEUs decreases. Therefore, in this case, the average capacity of the CCU increases while that of the CEU slight reduces.

When SINR threshold increases, more users with higher SINRs are classified as CEUs that can improve the average data rate of the CEU user. However, this improvement may trade-off with the increase in InterCell Interference when more users are served as CEUs. Therefore, it is observed that when
SINR threshold is greater than $12 \mathrm{~dB}$, the average capacity of the CEU remains fairly constant at $1.313(\mathrm{bit} / \mathrm{s} / \mathrm{Hz})$.
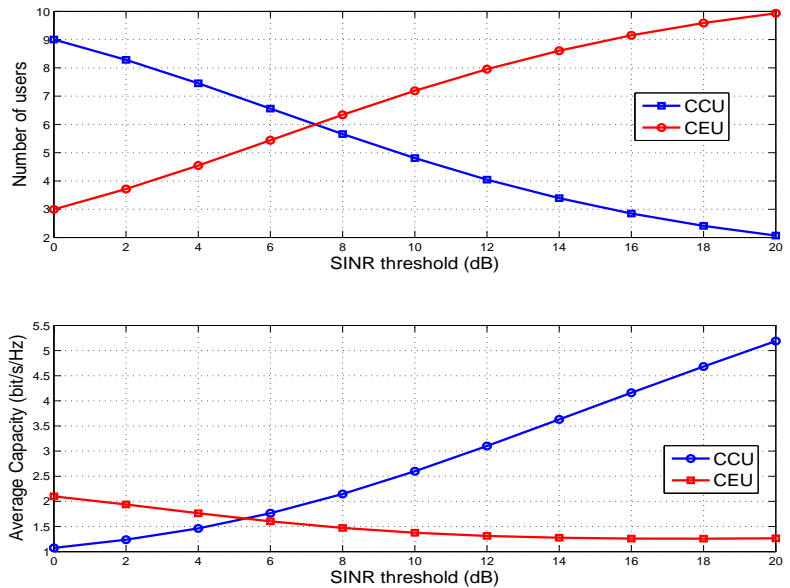

Fig. 5: Average number of CCUs and CCUs and Average data rate of the $\mathrm{CCU}$ and $\mathrm{CEU}$

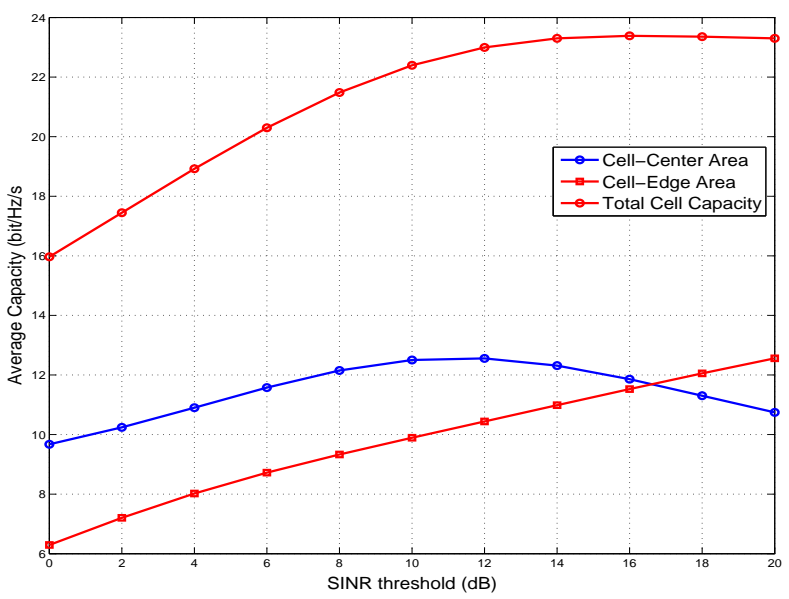

Fig. 6: Average data rate of Cell-Center and Cell-Edge Area

It is observed from Figure 6 that while the average capacity of the CE Area increase steadily, that of the CC Area rises to the peak of $12.3(\mathrm{bit} / \mathrm{s} / \mathrm{Hz})$ before moderately decreasing. Hence, the optimal SINR threshold can be selected at $14 \mathrm{~dB}$ when the average capacity of the cell peak at about 23.6 (bit/s/Hz).

\section{CONCLUSION}

In this paper, optimal value of SNR and SINR threshold are analysed through the performance of the CEU and CCU in a PPP network using Strict FR algorithm. The operation of the network is divided into two phases in which the users are classified as CCUs and CEUs during the first phase and the data transmission takes place during the second phase. The performance of the user is defined as the conditional probability of the second phase given the first phase. It is shown from the analytical results that a increase in SNR or SINR threshold results in the changes of number of CCUs 
and CEUs. At low-SNR or low-SINR threshold, the network obtains a balance between the number of CCUs and CEUs, and high network performance can be achieved. Meanwhile at high-SNR or high-SINR threshold, more users are classified as CEU that leads to the overload at CC Area and reduces the performance. Therefore, the optimal SNR and SINR threshold can be selected at 4 and $12 \mathrm{~dB}$, respectively.

\section{REFERENCES}

[1] A. S. Hamza, S. S. Khalifa, H. S. Hamza, and K. Elsayed, "A Survey on Inter-Cell Interference Coordination Techniques in OFDMA-Based Cellular Networks," IEEE Communications Surveys \& Tutorials, vol. 15, no. 4, pp. 1642-1670, 2013.

[2] J. G. Andrews, F. Baccelli, and R. K. Ganti, "A new tractable model for cellular coverage," in 2010 48th Annual Allerton Conference on Communication, Control, and Computing (Allerton), Conference Proceedings, pp. 1204-1211.

[3] H. ElSawy, E. Hossain, and M. Haenggi, "Stochastic Geometry for Modeling, Analysis, and Design of Multi-Tier and Cognitive Cellular Wireless Networks: A Survey," IEEE Communications Surveys Tutorials, vol. 15, no. 3, pp. 996-1019, Third 2013.

[4] M. Haenggi, Stochastic Geometry for Wireless Networks . Cambridge Univ. Press, November 2012

[5] F. Baccelli, M. Klein, M. Lebourges, and S. Zuyev, "Stochastic geometry and architecture of communication networks," Telecommunication Systems, vol. 7, no. 1, pp. 209-227, 1997. [Online]. Available: http://dx.doi.org/10.1023/A:1019172312328

[6] T. D. Novlan, R. K. Ganti, A. Ghosh, and J. G. Andrews, "Analytical Evaluation of Fractional Frequency Reuse for OFDMA Cellular Networks," IEEE Transactions on Wireless Communications, vol. 10, pp. 4294-4305, 2011.

[7] W. Bao and B. Liang, "Structured spectrum allocation and user association in heterogeneous cellular networks," in 2014 Proceedings of IEEE INFOCOM, April 2014, pp. 1069-1077.

[8] S. Kumar, S. Kalyani, and K. Giridhar, "Optimal design parameters for coverage probability in fractional frequency reuse and soft frequency reuse," IET Communications, vol. 9, no. 10, pp. 1324-1331, 2015.

[9] H. Zhuang and T. Ohtsuki, "A model based on Poisson point process for downlink K tiers fractional frequency reuse heterogeneous networks," Physical Communication, vol. Volume 13, Part B, no. Special Issue on Heterogeneous and Small Cell Networks, p. 312, 2014.

[10] S. C. Lam, R. Subramanian, K. Sandrasegaran, P. Ghosal, and S. Barua, "Performance of well-known frequency reuse algorithms in LTE downlink 3GPP LTE systems," in 2015 9th International Conference on Signal Processing and Communication Systems (ICSPCS), Dec 2015, pp. 1-5.

[11] W. S. K. J. M. Sung Nok Chiu, Dietrich Stoyan, Stochastic Geometry and Its Applications, 3rd ed. Wiley, 2013.

[12] S. C. Lam, R. Heidary, and K. Sandrasegaran, "A closed-form expression for coverage probability of random cellular network in composite Rayleigh-Lognormal fading channels," in 2015 International Telecommunication Networks and Applications Conference (ITNAC), 2015, pp. $161-165$. 